\title{
Radiological adjacent-segment degeneration in L4-5 spondylolisthesis: comparison between dynamic stabilization and minimally invasive transforaminal lumbar interbody fusion
}

\author{
${ }^{*}$ Chao-Hung Kuo, MD, ${ }^{1-3}$ Wen-Cheng Huang, MD, PhD, ${ }^{1,2}$ Jau-Ching Wu, MD, PhD, ${ }^{1,2}$ \\ Tsung-Hsi Tu, MD, , ${ }^{1,2,4}$ Li-Yu Fay, MD, , 1,2,5 Ching-Lan Wu, MD, ${ }^{2,6}$ and Henrich Cheng, MD, PhD ${ }^{1,2,5}$

\begin{abstract}
'Department of Neurosurgery, Neurological Institute, Taipei Veterans General Hospital; ${ }^{2}$ School of Medicine, National Yang-Ming University, Taipei, Taiwan; ${ }^{3}$ Department of Neurological Surgery, University of Washington, Seattle, Washington; ${ }^{4}$ Molecular Medicine Program, Taiwan International Graduate Program (TIGP), Academia Sinica; ${ }^{5}$ nnstitute of Pharmacology, National YangMing University; and ${ }^{6}$ Department of Radiology, Taipei Veterans General Hospital, Taipei, Taiwan
\end{abstract}

\begin{abstract}
OBJECTIVE Pedicle screw-based dynamic stabilization has been an alternative to conventional lumbar fusion for the surgical management of low-grade spondylolisthesis. However, the true effect of dynamic stabilization on adjacentsegment degeneration (ASD) remains undetermined. Authors of this study aimed to investigate the incidence of ASD and to compare the clinical outcomes of dynamic stabilization and minimally invasive transforaminal lumbar interbody fusion (Ml-TLIF).
\end{abstract}

METHODS The records of consecutive patients with Meyerding grade I degenerative spondylolisthesis who had undergone surgical management at L4-5 in the period from 2007 to 2014 were retrospectively reviewed. Patients were divided into two groups according to the surgery performed: Dynesys dynamic stabilization (DDS) group and MI-TLIF group. Pre- and postoperative radiological evaluations, including radiography, CT, and MRI studies, were compared. Adjacent discs were evaluated using 4 radiological parameters: instability (antero- or retrolisthesis), disc degeneration (Pfirrmann classification), endplate degeneration (Modic classification), and range of motion (ROM). Clinical outcomes, measured with the visual analog scale (VAS) for back and leg pain, the Oswestry Disability Index (ODI), and the Japanese Orthopaedic Association (JOA) scores, were also compared.

RESULTS A total of 79 patients with L4-5 degenerative spondylolisthesis were included in the analysis. During a mean follow-up of 35.2 months (range 24-89 months), there were 56 patients in the DDS group and 23 in the MI-TLIF group. Prior to surgery, both groups were very similar in demographic, radiological, and clinical data. Postoperation, both groups had similarly significant improvement in clinical outcomes (VAS, ODI, and JOA scores) at each time point of evaluation. There was a lower chance of disc degeneration (Pfirrmann classification) of the adjacent discs in the DDS group than in the MI-TLIF group (17\% vs 37\%, $p=0.01$ ). However, the DDS and MI-TLIF groups had similar rates of instability $(15.2 \%$ vs $17.4 \%$, respectively, $p=0.92)$ and endplate degeneration $(1.8 \% \mathrm{vs} 6.5 \%, p=0.30)$ at the cranial $(\mathrm{L} 3-4)$ and caudal (L5-S1) adjacent levels after surgery. The mean ROM in the cranial and caudal levels was also similar in the two groups. None of the patients required secondary surgery for any ASD (defined by radiological criteria).

CONCLUSIONS The clinical improvements after DDS were similar to those following MI-TLIF for L4-5 Meyerding grade I degenerative spondylolisthesis at 3 years postoperation. According to radiological evaluations, there was a lower chance of disc degeneration in the adjacent levels of the patients who had undergone DDS. However, other radiological signs of ASD, including instability, endplate degeneration, and ROM, were similar between the two groups. Although

\footnotetext{
ABBREVIATIONS ASD = adjacent-segment degeneration; DDD = degenerative disc disease; DDS = Dynesys dynamic stabilization; JOA = Japanese Orthopaedic Association; MI-TLIF = minimally invasive transforaminal lumbar interbody fusion; ODI = Oswestry Disability Index; ROM = range of motion; VAS = visual analog scale. SUBMITTED September 8, 2017. ACCEPTED January 11, 2018.

INCLUDE WHEN CITING Published online June 1, 2018; DOI: 10.3171/2018.1.SPINE17993.

* $\mathrm{CH}$ Kuo and WC Huang contributed equally to this paper.
} 
none of the patients in the present series required secondary surgery, a longer follow-up and a larger number of patients would be necessary to corroborate the protective effect of DDS against ASD.

https://thejns.org/doi/abs/10.3171/2018.1.SPINE17993

KEYWORDS ASD; Dynesys; TLIF; minimally invasive; spondylolisthesis; lumbar; degenerative

I NTERBODY fusion over L4-5 is the most commonly performed spinal arthrodesis procedure to treat symptomatic degenerative spondylolisthesis that is refractory to medical treatment or physical therapy. ${ }^{15,23}$ Surgical decompression, along with various interbody fusion techniques, such as the anterior, lateral, posterior, or transforaminal approaches, ${ }^{1,30}$ was considered a reasonable option for standard treatment of patients in whom conservative management had failed. In the last 2 decades, numerous reports have demonstrated the effectiveness of minimally invasive transforaminal lumbar interbody fusion (MITLIF), which can reduce blood loss and minimize soft tissue dissection during surgery with satisfactory clinical outcomes. $17,22,29,32$ On the other hand, Dynesys dynamic stabilization (DDS; Zimmer Biomet) has also proven viable for lumbar degenerative disc disease (DDD) $4,11,34,37$ and spondylolisthesis. ${ }^{5,7,823,28}$ In fact, several published reports have demonstrated that the two strategies, MI-TLIF and DDS, have had similar clinical and functional outcomes for patients with low-grade spondylolisthesis.,11-13 However, few reports have addressed the true incidence of adjacent-segment degeneration (ASD) after these spinal surgeries.

The pathological process observed at the spinal disc adjacent to the level that was treated with arthrodesis is regarded as ASD. Owing to advances in image technology, asymptomatic ASD can be identified by abnormal findings on radiological examinations; these findings include disc degeneration, segmental instability, or spinal stenosis and are termed "radiological ASD." These radiological ASDs can be completely free of symptoms or signs. However, there is also ASD that causes symptoms, such as back pain, leg pain, or claudication. Adjacent disc abnormalities found radiologically that do correlate with clinical symptoms are referred to as "clinical ASD." 20

The risk factors and mechanisms of ASD have been widely discussed but never clearly defined. , $^{8,16,20,36,38} \mathrm{Al}$ though some reports have claimed that the strategy of motion preservation rather than spinal fusion (e.g., DDS) has the potential for ameliorating ASD, its true effect on the adjacent disc has remained undetermined. Therefore, in this study, we aimed to compare the clinical and radiological outcomes between two groups of surgical patients (MITLIF and DDS). Given the low incidence of clinical ASD, this study specifically focused more on radiological ASD.

\section{Methods \\ Patient Population}

Consecutive patients who had undergone single-level surgical instrumentation at L4-5 at a single medical center (Taipei Veteran's General Hospital) in the period from 2007 to 2014 were potentially eligible for inclusion in this study. All clinical records, radiological data, and clinical evaluations for these patients were retrospectively reviewed. Study inclusion criteria were symptomatic L4-5 DDD or stenosis with Meyerding grade I spondylolisthesis that was refractory to nonsurgical management for at least 12 weeks. Exclusion criteria were multiple levels of DDD, Meyerding grade II (or higher) spondylolisthesis, degenerative scoliosis, a history of connective tissue diseases, vertebral fracture, infection, tumor, inability to cooperate with the study, or loss during a minimum of 2 years' follow-up. All patients were divided into two groups according to their treatment: DDS and MI-TLIF groups. The choice of DDS or MI-TLIF was made by a consensus reached after a conference led by the attending surgeon to discuss the issue with the patient and their family. Because of the retrospective nonrandomized design of this study, modest selection bias could inevitably exist. This issue is addressed as one of the study's limitations in the Discussion. All pre- and postoperative clinical and radiological outcome evaluations were analyzed.

\section{Surgical Techniques}

All patients underwent general anesthesia and were placed prone with the lumbar spine in a neutral or slightly lordotic position.

For patients of the DDS group, who underwent dynamic pedicle screw insertion using the Dynesys system, an L-4 total laminectomy was performed via a posterior midline incision. Subsequently, bilateral neural foramen and lateral recesses were decompressed with Kerrison rongeurs. Bilateral facet joints were carefully preserved. After subdermal dissection through the same midline skin incision, the Dynesys titanium alloy screws were placed transpedicularly through the Wiltse plane. The elastic rods, polycarbonate urethane spacers, and polyethyleneterephthalate cords (Sulene-PET) were assembled without intentional intraoperative reduction of any listhesis or change of the lordotic curvature. The procedure involved the use of lateral fluoroscopy periodically for confirmation of optimal implant positions.

For patients of the MI-TLIF group, tubular retractors were placed, after the serial dilators, on the indexed facet joint under fluoroscopic guidance. Under microscopic guidance, facetectomy and partial laminectomy were performed with high-speed drills and Kerrison rongeurs. Similar procedures were done for the contralateral side decompression. After resection of the hypertrophic ligamentum flavum and discectomy, local bone autografts and demineralized bone matrix were packed into the disc space. Then a polyetheretherketone (PEEK) interbody cage was placed into the disc space via Kambin's triangle. After removal of the tubular retractors, the percutaneous placement of four pedicle screws was undertaken under intraoperative fluoroscopy. 
All wounds were subsequently closed in layers after copious irrigation.

\section{Clinical and Radiological Evaluations of ASD}

Clinical outcomes were measured using the visual analog scale (VAS) for back and leg pain, the Oswestry Disability Index (ODI), and the Japanese Orthopaedic Association (JOA) clinical symptom scores. Questionnaires were completed preoperatively and at approximately 6,12 , 18 , and 24 months postoperatively by the patients with the assistance of a special nurse and the supervision of the physician during each clinic visit.

Radiological evaluation of the adjacent discs utilized multiple modalities, including radiography, CT, and MRI. The CT scans were obtained preoperatively, within 5 days postoperatively, and 12-24 months postoperatively. Every patient underwent MRI preoperatively, which was used as the baseline comparison in the study, and approximately annually in the postoperative follow-up period. Radiological ASD was measured according to the following abnormal image findings: listhesis (antero- or retrolisthesis), instability, herniated or degenerative disc, hypertrophic facet joints, and spinal stenosis. ${ }^{21}$ Pre- and postoperative images were evaluated and compared by neurosurgeons and radiologists. The definition of segmental instability included vertebral slippage on neutral lateral radiographs with increased angular motion on lateral flexion and extension radiographs. Postoperative CTs were used for the detection of screw malpositioning or loosening. Postoperative MRI was used for the evaluation of stenosis and disc and endplate degeneration, according to the Pfirrmann classification (grades I-V) and the modified Modic classification. ${ }^{6,18,24,27}$ The latter classification is as follows: 0 (normal), 1 (Modic type I: pure edema endplate signal changes), 2 (Modic type I-2: mixed edema and fatty changes, predominantly edema), 3 (Modic type II-1: mixed changes, predominantly fatty), and 4 (Modic type II: pure fatty endplate changes). All of the radiological evaluations were conducted by Drs. Kuo and CL Wu independently, and consensus was reached by discussion among all the authors if there were any inconsistencies.

\section{Statistical Analysis}

Data are presented as the mean \pm standard deviation for continuous variables and as frequency and percentage for categorical variables. All statistical tests were two-tailed, and a $\mathrm{p}<0.05$ was considered statistically significant by independent t-test, paired t-test, or chi-square test. All statistical analyses were performed using MedCalc software.

\section{Results}

A total of 129 patients (98 DDS group vs 31 MI-TLIF group) with L4-5 Meyerding grade I degenerative spondylolisthesis and spinal stenosis, confirmed by lateral radiographs, CT scans, and MR images preoperatively, underwent surgical treatment. Both types of surgeries were performed by Drs. JC Wu, Huang, and Cheng. Seventynine patients completed more than 2 years of follow-up (mean 35.2 months, range 24-89 months). For the retrospective analysis, patients were grouped into two catego-
TABLE 1. Preoperative evaluations and demographics

\begin{tabular}{lccc}
\hline \multirow{2}{*}{ Characteristic } & \multicolumn{2}{c}{ Group } & \multirow{2}{p}{} \\
\cline { 2 - 3 } & DDS & Ml-TLIF & Value \\
\hline No. of patients & 56 & 23 & \\
\hline Age in yrs & $60.1 \pm 10.8$ & $58.0 \pm 11.7$ & 0.44 \\
\hline Sex (F/M) & $35 / 21$ & $15 / 8$ & 0.98 \\
\hline BMI & $24.5 \pm 2.9$ & $26.2 \pm 4.4$ & 0.53 \\
\hline Diabetes mellitus & $8(14.3 \%)$ & $5(21.7 \%)$ & 0.63 \\
\hline Smoking & $3(5.4 \%)$ & $4(17.4 \%)$ & 0.20 \\
\hline Hypertension & $19(33.9 \%)$ & $9(39.1 \%)$ & 0.86 \\
\hline Preop symptoms & & & \\
\hline Neurogenic claudication & $35(62.5 \%)$ & $16(69.6 \%)$ & 0.74 \\
\hline Radicular pain & $55(98.2 \%)$ & $23(100 \%)$ & 0.64 \\
\hline Preop evaluations & & & \\
\hline VAS back pain & $6.1 \pm 3.0$ & $6.7 \pm 2.1$ & 0.42 \\
\hline VAS leg pain & $7.1 \pm 2.4$ & $6.0 \pm 3.0$ & 0.18 \\
\hline ODI & $24.7 \pm 9.2$ & $23.3 \pm 7.8$ & 0.50 \\
\hline JOA & $4.6 \pm 3.1$ & $5.3 \pm 3.0$ & 0.48 \\
\hline Preop angular motion in degrees & & & \\
(lat flexion-extension) & & & \\
\hline L3-4 & $3.5 \pm 5.3$ & $3.7 \pm 4.4$ & 0.15 \\
\hline L4-5 & $5.8 \pm 5.2$ & $4.5 \pm 4.2$ & 0.28 \\
\hline L5-S1 & $5.1 \pm 4.5$ & $3.7 \pm 4.4$ & 0.21 \\
\hline V-5 & & & \\
\hline
\end{tabular}

Values are expressed as the mean \pm standard deviation or as frequency (\%), unless indicated otherwise.

ries according to the surgical strategy used (DDS and MITLIF groups).

In the DDS group, there were 56 patients $(71 \%)$ who had undergone standard open laminectomy and insertion of Dynesys dynamic pedicle screws. In the MI-TLIF group, there were 23 patients $(29 \%)$ who had undergone MI-TLIF with decompression and interbody grafting via tubular retractors and then percutaneous pedicle screw placement. There were no significant statistical differences in the demographic data between the DDS and MI-TLIF groups, including patient age, sex, BMI ( $\mathrm{p}=0.44,0.98$, and 0.53 , respectively), and comorbidity, such as diabetes mellitus, cigarette smoking, and hypertension $(\mathrm{p}=0.63$, 0.20 , and 0.86 , respectively; Table 1). The preoperative clinical symptoms of neurogenic claudication or radicular pain were similar between the DDS and MI-TLIF groups. Also, there were no differences between the two groups in the preoperative VAS back pain, VAS leg pain, ODI, and JOA scores.

\section{Clinical Outcomes}

After surgery, both groups had significant clinical improvement according to VAS back pain, VAS leg pain, ODI, and JOA scores, compared to the preoperative scores. Furthermore, there were no differences between the two groups. The mean VAS back pain score significantly improved from $6.1 \pm 3.0$ to $2.3 \pm 2.3$ and from $6.7 \pm$ 2.1 to $2.3 \pm 2.1$ in the DDS and MI-TLIF groups, respectively (both $\mathrm{p}<0.001$ ). The mean VAS leg pain score also 
TABLE 2. Postoperative clinical characteristics

\begin{tabular}{|c|c|c|c|}
\hline \multirow[b]{2}{*}{ Characteristic } & \multicolumn{2}{|c|}{ Group } & \multirow{2}{*}{$\begin{array}{c}\mathrm{p} \\
\text { Value }\end{array}$} \\
\hline & DDS & MI-TLIF & \\
\hline No. of segments & 112 & 46 & \\
\hline \multicolumn{4}{|l|}{ 2-yr postop evaluations } \\
\hline VAS back pain & $2.3 \pm 2.3$ & $2.3 \pm 2.1$ & 0.22 \\
\hline VAS leg pain & $2.3 \pm 2.3$ & $1.6 \pm 1.9$ & 0.91 \\
\hline ODI & $10.8 \pm 7.6$ & $8.8 \pm 6.6$ & 0.74 \\
\hline $\mathrm{JOA}$ & $9.9 \pm 3.2$ & $10.2 \pm 3.5$ & 0.76 \\
\hline Mean FU in mos & 34.7 & 36.3 & 0.73 \\
\hline $\begin{array}{l}\text { Disc degeneration (Pfirrmann } \\
\text { classification) }\end{array}$ & $19(17.0 \%)$ & $17(37.0 \%)$ & 0.01 \\
\hline Instability (antero- or retrolisthesis) & $17(15.2 \%)$ & $8(17.4 \%)$ & 0.92 \\
\hline $\begin{array}{l}\text { Endplate degeneration (Modic } \\
\text { classification) }\end{array}$ & $2(1.8 \%)$ & $3(6.5 \%)$ & 0.30 \\
\hline \multicolumn{4}{|l|}{$\begin{array}{l}\text { Postop angular motion in degrees } \\
\text { (lat flexion-extension) }\end{array}$} \\
\hline L3-4 & $4.6 \pm 3.9$ & $3.3 \pm 3.7$ & 0.15 \\
\hline L4-5 & $1.4 \pm 2.5$ & $0.0 \pm 1.1$ & $<0.01$ \\
\hline L5-S1 & $4.1 \pm 4.3$ & $3.3 \pm 4.2$ & 0.45 \\
\hline Secondary surgery (\%) & 0 & 0 & \\
\hline
\end{tabular}

FU = follow-up.

Values expressed as the mean \pm standard deviation or as frequency (\%), unless indicated otherwise. Boldface type indicates statistical significance.

improved significantly from $7.1 \pm 2.4$ to $2.3 \pm 2.3$ and from $6.0 \pm 3.0$ to $1.6 \pm 1.9$ in the DDS and MI-TLIF groups, respectively (both $\mathrm{p}<0.001$ ). Furthermore, at each time point of the postoperative evaluation, there were no differences in VAS scores between the two groups (Tables 1 and 2 and Figs. 1 and 2).

Both the DDS and MI-TLIF groups had similar improvement in the ODI and JOA scores after surgery. From preoperation to 2 years postoperation, the ODI improved from $24.7 \pm 9.2$ to $10.8 \pm 7.6$ and $23.3 \pm 7.8$ to $8.8 \pm 6.6$ in the DDS and MI-TLIF groups, respectively (both $\mathrm{p}<$ $0.001)$. In the same period, the JOA score improved from $4.6 \pm 3.1$ to $9.9 \pm 3.2$ and $5.3 \pm 3.0$ to $10.2 \pm 3.5$ in the DDS

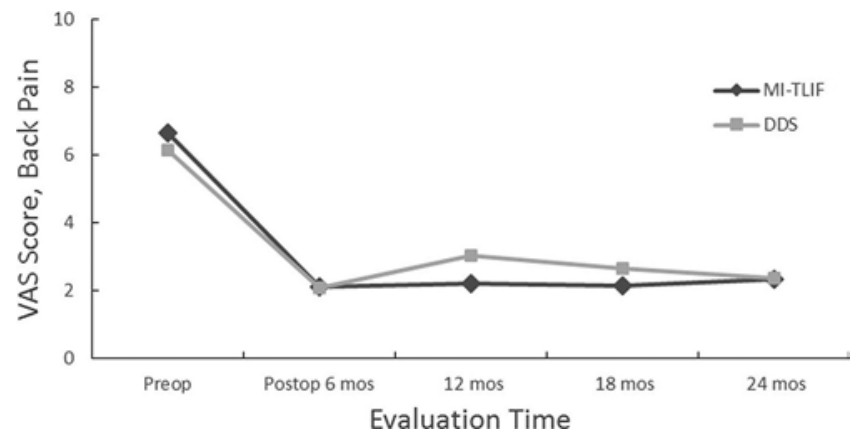

FIG. 1. Clinical outcomes measured according to the average VAS back pain score, with significant improvement postoperatively but without significant difference between the MI-TLIF and DDS groups at different time points.

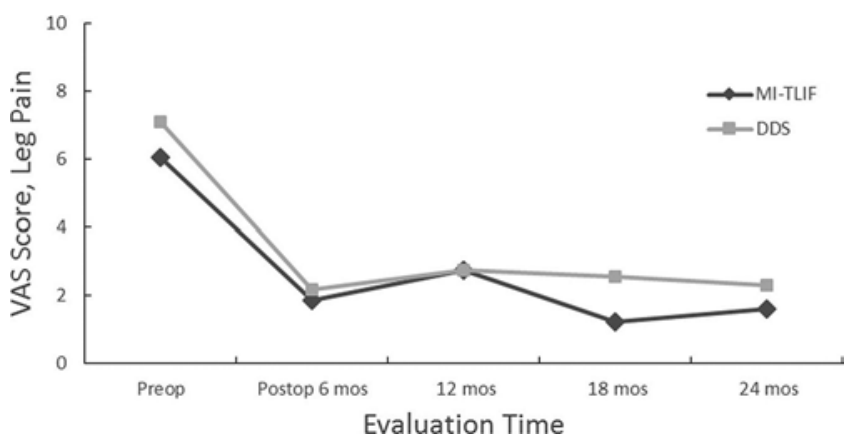

FIG. 2. Clinical outcomes measured according to the average VAS leg pain score, demonstrating significant improvement postoperatively. There were no significant differences between the MI-TLIF and DDS groups at each time point.

and MI-TLIF groups, respectively (both $\mathrm{p}<0.001$ ). Furthermore, there were no inter- or intragroup differences at each evaluation time point (Tables 1 and 2 and Figs. 3 and 4).

\section{Radiological Outcomes}

Per all diagnostic criteria, during a follow-up of more than 2 years, $41(51.9 \%)$ patients had radiological ASD, 15 $(65.2 \%)$ in the MI-TLIF group and $26(46.4 \%)$ in the DDS group $(\mathrm{p}=0.13)$.

Specifically looking into disc degeneration on MRI and using the Pfirrmann classification, we found a higher incidence in the MI-TLIF group than in the DDS group (37.0\% vs $17.0 \%, \mathrm{p}=0.01$; Table 2$)$. The pre- and postoperative evaluations of each adjacent disc according to the Pfirrmann classification for both groups are illustrated in Tables 3 and 4.

The DDS group had a tendency toward less ASD according to the other three parameters of postoperative listhesis, endplate condition, and angular motion of adjacent discs, compared to that in the MI-TLIF group. However, the differences did not reach statistical significance. Postoperative antero- and retrolisthesis, evaluated on lateral dynamic radiographs, were a little less in the DDS group than in the MI-TLIF group (15.2\% vs $17.4 \%, \mathrm{p}=0.92$; Ta-

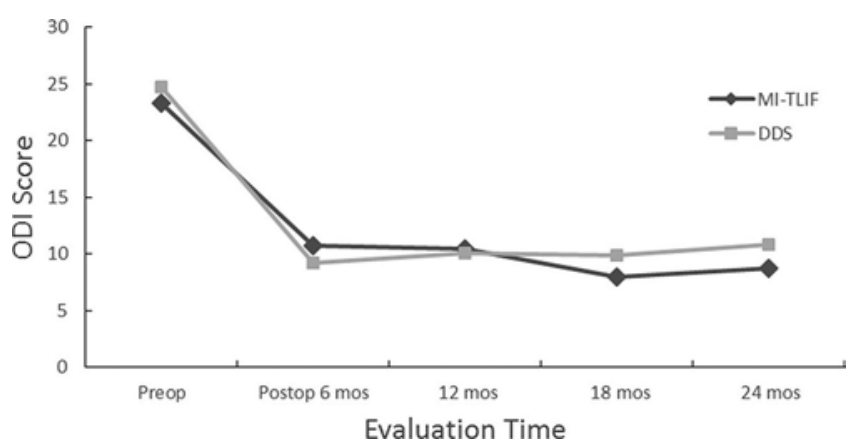

FIG. 3. Clinical outcomes measured according to the ODI, demonstrating significant improvement postoperatively. There were no significant differences between the MI-TLIF and DDS groups at each time point. 


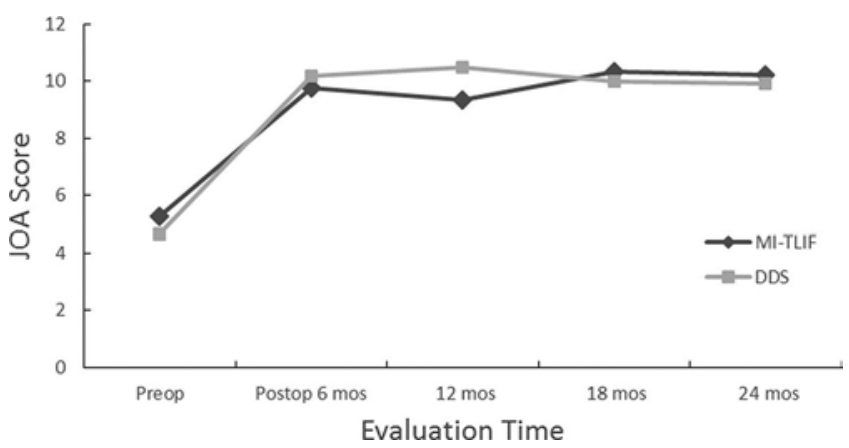

FIG. 4. Clinical outcomes measured according to the JOA scores, demonstrating significant improvement postoperatively. There were no significant differences between the MI-TLIF and DDS groups at each time point.

ble 2). Endplate degeneration, evaluated according to the Modic classification, was also less in the DDS group, but the difference did not reach statistical significance $(1.8 \%$ vs $6.5 \%, \mathrm{p}=0.30$; Table 2). Detailed comparisons of preand postoperative changes are illustrated in Tables 5 and 6.

Patients who underwent DDS demonstrated a modestly preserved range of motion $\left(\mathrm{ROM} ; 1.4^{\circ} \pm 2.5^{\circ}\right)$ at $\mathrm{L} 4-5$ postoperatively, the index level, which was significantly larger than that in the MI-TLIF group. Preoperatively, there was little difference between the groups for the index and adjacent levels (Table 1). Postoperatively, there were also no differences for the adjacent levels, either L3-4 or L5-S1 (all p > 0.05; Table 2). Furthermore, the intragroup comparison also demonstrated little difference pre- and postoperation except at the index level (Table 7). Therefore, both DDS and MI-TLIF had little influence on the postoperative ROM of adjacent disc levels, L3-4 and L5-S1, among patients in this series.

During the follow-up, no patient underwent a secondary or revision surgery for ASD.

\section{Illustrative Cases \\ Case 1}

A 48-year-old female had low-back pain and bilateral leg pain for years, which had not responded to conservative treatment. The preoperative MRI studies demonstrated spinal stenosis at L4-5 caused by both bulging annulus

TABLE 3. Pre- and postoperative Pfirrmann classification of cranial and caudal adjacent levels in patients who underwent MI-TLIF

\begin{tabular}{crrrrr}
\hline & \multicolumn{5}{c}{ Postop Grade } \\
\cline { 2 - 6 } Preop Grade & I & II & III & IV & V \\
\hline I & 0 & 0 & 0 & 0 & 0 \\
\hline II & 0 & 5 & 3 & 2 & 0 \\
\hline III & 0 & 0 & 12 & 9 & 1 \\
\hline IV & 0 & 0 & 0 & 9 & 2 \\
\hline V & 0 & 0 & 0 & 0 & 3 \\
\hline
\end{tabular}

TABLE 4. Pre- and postoperative Pfirrmann classification of cranial and caudal adjacent levels in patients who underwent DDS

\begin{tabular}{crrrrr}
\hline & \multicolumn{5}{c}{ Postop Grade } \\
\cline { 2 - 6 } Preop Grade & I & II & III & IV & V \\
\hline I & 0 & 0 & 0 & 0 & 0 \\
\hline II & 0 & 10 & 3 & 0 & 0 \\
\hline III & 0 & 0 & 49 & 9 & 2 \\
\hline IV & 0 & 0 & 0 & 29 & 5 \\
\hline V & 0 & 0 & 0 & 0 & 5 \\
\hline
\end{tabular}

fibrosis and hypertrophic ligamentum flavum (Fig. 5A and C) with Meyerding grade I degenerative spondylolisthesis (Fig. 5E). She underwent L-4 total laminectomy and DDS at L4-5. Her symptoms remarkably improved after surgery (Fig. 5B). At 2 years postoperation, MRI demonstrated no cranial- or caudal-level adjacent disc or endplate degeneration (Fig. 5D), and radiographs revealed that the DDS remained as a stable construct without any retrolisthesis at adjacent levels (Fig. 5F).

\section{Case 2}

A 58-year-old female had low-back pain and bilateral leg pain for years. Moreover, there had been progressive neurogenic claudication for 6 months prior to her presentation. Her preoperative MRI studies demonstrated spinal stenosis caused by bulging annular fibrosis and hypertrophic ligamentum flavum and facet arthropathies at L4-5 (Fig. 6A). Lateral dynamic radiographs demonstrated Meyerding grade I spondylolisthesis at L4-5 (Fig. $6 \mathrm{E})$. Given that her symptoms were refractory to medical management and her claudication had rapidly deteriorated, she underwent surgery. Minimally invasive TLIF was performed, and there were no adverse events postoperatively. The patient's symptoms improved significantly after surgery (Fig. 6B). At the 2-year radiological follow-up, MRI demonstrated disc degeneration over the cranial and caudal adjacent levels, as compared to that preoperatively (Fig. 6D; L3-4 from Pfirrmann grade II to grade IV; L5S1 from Pfirrmann grade II to grade IV). Lateral dynamic radiographs also demonstrated L5-S1 listhesis (Fig. 6F). The patient had few symptoms during continued followup, without any secondary surgery to date.

TABLE 5. Pre- and postoperative Modic classification of cranial and caudal adjacent levels in patients who underwent MI-TLIF

\begin{tabular}{crrrrr}
\hline & \multicolumn{5}{c}{ Postop Grade } \\
\cline { 2 - 6 } Preop Grade & 0 & 1 & 2 & 3 & 4 \\
\hline 0 & 40 & 2 & 1 & 0 & 0 \\
\hline 1 & 0 & 0 & 0 & 0 & 0 \\
\hline 2 & 0 & 0 & 3 & 0 & 0 \\
\hline 3 & 0 & 0 & 0 & 0 & 0 \\
\hline 4 & 0 & 0 & 0 & 0 & 0 \\
\hline
\end{tabular}


TABLE 6. Pre- and postoperative Modic classification of cranial and caudal adjacent levels in patients who underwent DDS

\begin{tabular}{crrrrr}
\hline & \multicolumn{5}{c}{ Postop Grade } \\
\cline { 2 - 6 } Preop Grade & 0 & 1 & 2 & 3 & 4 \\
\hline 0 & 103 & 0 & 0 & 0 & 0 \\
\hline 1 & 0 & 0 & 1 & 0 & 0 \\
\hline 2 & 0 & 0 & 7 & 1 & 0 \\
\hline 3 & 0 & 0 & 0 & 0 & 0 \\
\hline 4 & 0 & 0 & 0 & 0 & 0 \\
\hline
\end{tabular}

\section{Discussion}

The present study was the first to address the differences in radiological ASD after DDS or MI-TLIF surgery. We specifically focused on symptomatic one-level Meyerding grade I degenerative spondylolisthesis and attempted to correlate the radiological findings with clinical outcomes. Only patients with L4 -5 spondylolisthesis and spinal stenosis that were refractory to medical management were included in the study. A total of 79 patients were retrospectively divided into DDS or MI-TLIF groups (56 vs 23, respectively) and followed up for a mean of 35.2 months (range 24-89 months). Overall, there were postoperative improvements in neurological function and clinical outcomes (VAS, ODI, and JOA scores) in both groups. As regards radiological follow-up, the DDS group had a significantly lower incidence of adjacent disc degeneration than the MI-TLIF group. There was a higher incidence of radiological ASD in the MI-TLIF group than in the DSS group, but the difference between the two groups did not reach statistical significance. When combining all four criteria of radiological ASD (i.e., disc degeneration, instability, endplate degeneration, and mobility), the advantages of DDS for ASD seemed inconclusive. This finding could theoretically be attributed to the relatively low incidence rates of ASD in both the DDS and MI-TLIF groups at the midterm follow-up, such as in the present study. The benefits of DDS for protection against ASD may not be demonstrated until a later follow-up period.

For patients with symptomatic lumbar spondylolisthesis, conventional TLIF has been accepted as one of the options for surgical management. ${ }^{9}$ In past decades many published series have demonstrated similar clinical outcomes with MI-TLIF, which has caused less collateral tissue damage and blood loss, required a shorter hospital stay, and yielded an earlier recovery than conventional TLIF., 10,33 There was also the emerging option of dynamic stabilization in the management of low-grade spondylolisthesis with satisfactory results. The surgical approach of dynamic stabilization involves more soft tissue dissection than percutaneous pedicle screw insertion, but its biomechanical alterations remain elusive. These changes include increased stress and hypermobility of the cranial or caudal level adjacent to the fused segment, together with the aging process, which may play a primary role in causing ASD. ${ }^{21,25}$ There are also several risk factors that could potentiate ASD, such as long-segment fusion, ${ }^{26,35}$ preexisting disc degeneration at the adjacent level, ${ }^{19}$ older age, ${ }^{2,26}$ osteoporosis, ${ }^{2}$ intraoperative proximal facet violation, ${ }^{14}$ and higher BMI. ${ }^{14}$ In another retrospective study of 27 elderly patients with degenerative spondylolisthesis who had been treated with mini-open TLIF, ASD was found in 12 patients (44.4\%) without clinical symptoms at 38.6 months postoperation. ${ }^{16}$ In another retrospective study of patients treated with MI-TLIF, 11 (2.14\%) of 513 patients had postoperative adjacent degenerative disease. Very few studies have compared the incidence of ASD between open and MI-TLIF. Authors who retrospectively analyzed 68 patients (52 MI-TLIF vs 16 open TLIF) found adjacent-segment disease in 7 patients, 4 from the MI-TLIF group and 3 from the open TLIF group..$^{38}$ The study demonstrated little difference in the incidence of adjacent-segment disease between the two groups. Lau et al. also demonstrated that the incidence rate of intraoperative proximal facet violation was not different between MI-TLIF (142 patients) and open TLIF (140 patients) groups. ${ }^{14}$

A few series comparing the clinical outcomes between DDS and TLIF for the treatment of symptomatic spondylolisthesis have been published in the past decade and have demonstrated that dynamic stabilization with Dynesys pedicle screws is an acceptable alternative to fusion., ${ }^{3,11-13}$ It remains uncertain if DDS can lower the incidence of ASD based on the concept of nonfusion. In a study of 39 patients with degenerative lumbar spondylolisthesis at L4-5 treated with bilateral decompression and DDS, ASD was found in $28.2 \%$ of segments at the cranial level (L3-4) and $17.9 \%$ at the caudal level (L5-S1) without adverse clinical effects. ${ }^{8}$ It was also reported that among 19 patients who had symptomatic lumbar spinal stenosis and degenerative spondylolisthesis and who underwent DDS, 9 patients (47\%) developed ASD, 8 at the cranial adjacent

TABLE 7. Pre- and postoperative angular motion

\begin{tabular}{|c|c|c|c|c|c|c|}
\hline \multirow[b]{3}{*}{ Parameter } & \multicolumn{6}{|c|}{$\mathrm{ROM}^{*}$} \\
\hline & \multicolumn{3}{|c|}{ DDS } & \multicolumn{3}{|c|}{ MI-TLIF } \\
\hline & L3-4 & L4-5 & L5-S1 & L3-4 & L4-5 & L5-S1 \\
\hline Preop ROM & $5.3 \pm 3.5$ & $5.8 \pm 5.2$ & $5.1 \pm 4.5$ & $3.7 \pm 4.4$ & $4.5 \pm 4.2$ & $3.7 \pm 4.4$ \\
\hline Postop ROM & $4.6 \pm 3.9$ & $1.4 \pm 2.5$ & $4.1 \pm 4.3$ & $3.3 \pm 3.7$ & $0.0 \pm 1.1$ & $3.3 \pm 4.2$ \\
\hline$p$ value & 0.33 & $<0.01$ & 0.06 & 0.67 & $<0.01$ & 0.65 \\
\hline
\end{tabular}

Values are expressed as the mean \pm standard deviation in degrees, unless indicated otherwise.

* By angular difference between lateral flexion and extension. 


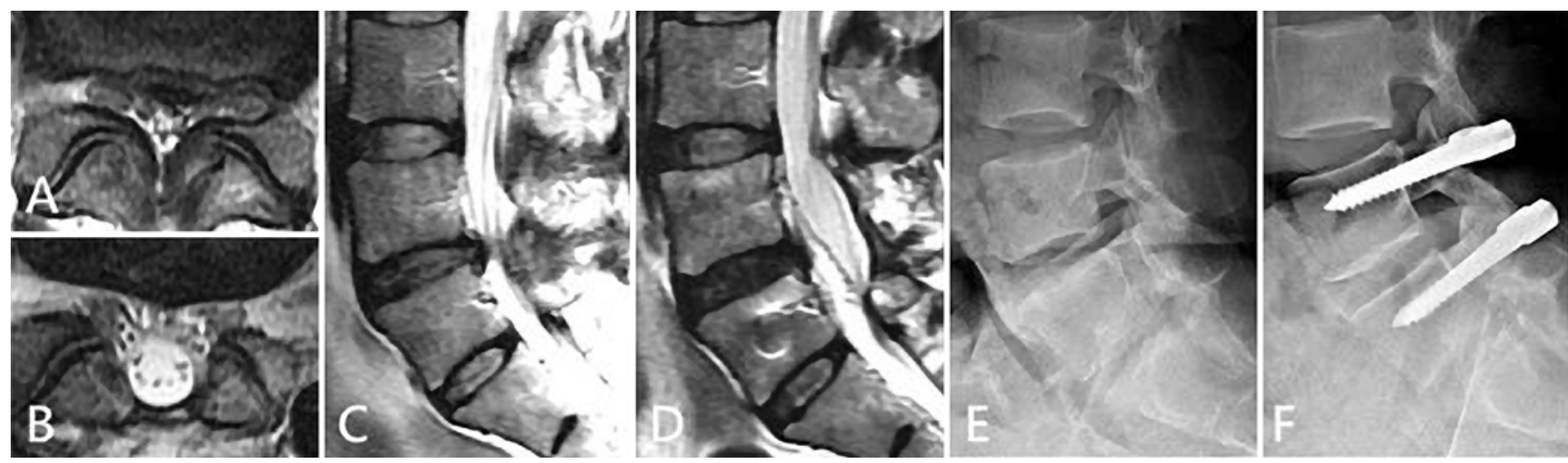

FIG. 5. A 48-year-old female had low-back pain and bilateral leg pain for years. Preoperative MRI demonstrated spinal stenosis at L4-5 caused by a bulging annulus fibrosis and hypertrophic ligamentum flavum ( $\mathbf{A}$ and $\mathbf{C}$ ) with Meyerding grade I spondylolisthesis (E). After L-4 laminectomy and DDS, she experienced symptom relief, and the 2-year follow-up images demonstrated a stable construct of the DDS without listhesis $(\mathbf{F})$ and little spinal stenosis or disc degeneration (B and D).

segment and 1 at the caudal adjacent segment. ${ }^{28}$ Another retrospective study analyzed 52 patients who had undergone DDS and identified $15(29 \%)$ patients with ASD at 4 -years' follow-up. ${ }^{31}$ However, none of these studies specifically compared DDS to TLIF. In the present study, the incidence rate of ASD in the MI-TLIF group was higher than that in the DDS group, although there was no statistical significant difference between the two groups. This observation was compatible with the common assumption that arthrodesis can accelerate ASD because of the shifted loading and increased mobility inevitably conveyed from the fused vertebral bodies. Nevertheless, the true effect of DDS on ASD may require very long observation and quite a large number of patients to detect, especially given the nature of ASD and the process of aging.

There are limitations to the current study. It was a retrospective nonrandomized cohort study. Patients were allocated to one of the treatments, DDS or MI-TLIF, based on the surgeons' decisions after thorough communication with each patient and their family. Although all patients should have received a clear explanation by the surgeons prior to surgery and the two groups appeared to be very similar in demographics (Table 1), there may have been some selection bias. Furthermore, neither the patients nor the surgeons were blinded to the choices of treatment. There were economic differences between the two treatments as well. Patients' out-of-pocket expenditures for the MI-TLIF surgery at our institute were approximately twice as high as those for the DDS group. This cost difference may also result in discrepancies in expectations of the surgical outcome and thus cause bias in self-reported evaluations. Nonetheless, the clinical outcomes were very similar for the two treatments during the follow-up period. The radiological outcomes, focusing on ASD, slightly favored DDS over MI-TLIF, supporting the theoretical effects of DDS on the deceleration of degeneration. However, because of the low incidence of ASD shortly after the operations, a long enough follow-up-for example, 10 to 15 years - may be necessary to differentiate the true effects of these two treatment modalities. Whether the ASD
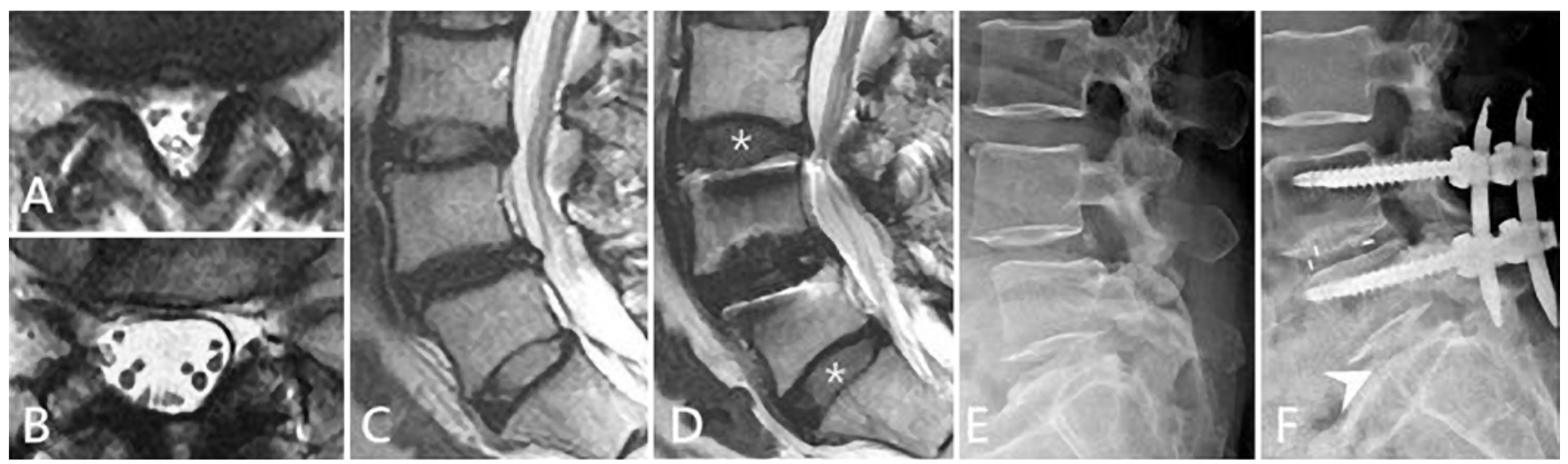

FIG. 6. A 58-year-old female presented with low-back pain, bilateral leg pain, and claudication. Preoperative MRI demonstrated spinal stenosis at L4-5 caused by a bulging disc and hypertrophic ligamentum flavum ( $A$ and $C$ ), and lateral radiography showed Meyerding grade I spondylolisthesis (E). She underwent MI-TLIF (B). Two-year follow-up MRI demonstrated disc degeneration over the cranial and caudal adjacent levels ( $D$, asterisks), and lateral radiography also revealed L5-S1 listhesis ( $F$, arrowhead). To date, the patient has remained free of symptoms and has been closely followed up. 
was device related or a natural course remains undetermined since the study did not have a control group that did not undergo surgery. Results of the current study obviously suggest the need for greater case numbers and a longer duration (at least 5 years) of observation to corroborate the clinical equivalence. The study had merit in narrowing down the scope of its investigation to only L4-5 spondylolisthesis. That meant the comparison of ASD only at the L3-4 cranial and L5-S1 caudal levels. Indeed, the present study shed light on the potential advantage of DDS, a pedicle screw-based dynamic stabilization device.

\section{Conclusions}

The clinical improvements following DDS were similar to those after MI-TLIF for L4-5 Meyerding grade I degenerative spondylolisthesis at almost 3 years postoperation. According to radiological evaluations, there was a lower chance of disc degeneration in the adjacent levels in the patients who underwent DDS. However, other radiological signs of ASD, including instability, endplate degeneration, and ROM, were similar between the two treatment groups. Although none of the patients required secondary surgery, a longer follow-up and an increased number of patients would be necessary to corroborate the protective effect of DDS against ASD.

\section{References}

1. Eismont FJ, Norton RP, Hirsch BP: Surgical management of lumbar degenerative spondylolisthesis. J Am Acad Orthop Surg 22:203-213, 2014

2. Etebar S, Cahill DW: Risk factors for adjacent-segment failure following lumbar fixation with rigid instrumentation for degenerative instability. J Neurosurg 90 (2 Suppl):163-169, 1999

3. Fay LY, Chang PY, Wu JC, Huang WC, Wang CH, Tsai TY, et al: Dynesys dynamic stabilization-related facet arthrodesis. Neurosurg Focus 40(1):E4, 2016

4. Fay LY, Wu JC, Tsai TY, Tu TH, Wu CL, Huang WC, et al: Intervertebral disc rehydration after lumbar dynamic stabilization: magnetic resonance image evaluation with a mean followup of four years. Adv Orthop 2013:437570, 2013

5. Fay LY, Wu JC, Tsai TY, Wu CL, Huang WC, Cheng H: Dynamic stabilization for degenerative spondylolisthesis: evaluation of radiographic and clinical outcomes. Clin Neurol Neurosurg 115:535-541, 2013

6. Fayad F, Lefevre-Colau MM, Drapé JL, Feydy A, Chemla N, Quintéro N, et al: Reliability of a modified Modic classification of bone marrow changes in lumbar spine MRI. Joint Bone Spine 76:286-289, 2009

7. Fayyazi AH, Ordway NR, Park SA, Fredrickson BE, Yonemura K, Yuan HA: Radiostereometric analysis of postoperative motion after application of dynesys dynamic posterior stabilization system for treatment of degenerative spondylolisthesis. J Spinal Disord Tech 23:236-241, 2010

8. Hoppe S, Schwarzenbach O, Aghayev E, Bonel H, Berlemann U: Long-term outcome after monosegmental L4/5 stabilization for degenerative spondylolisthesis with the Dynesys device. Clin Spine Surg 29:72-77, 2016

9. Jhala A, Singh D, Mistry M: Minimally invasive transforaminal lumbar interbody fusion: Results of 23 consecutive cases. Indian J Orthop 48:562-567, 2014

10. Kaloostian PE, Gokaslan ZL: Evidence-based review of transforaminal lumbar interbody fusion: is minimally invasive better? World Neurosurg 82:65-67, 2014
11. Ko CC, Tsai HW, Huang WC, Wu JC, Chen YC, Shih YH, et al: Screw loosening in the Dynesys stabilization system: radiographic evidence and effect on outcomes. Neurosurg Focus 28(6):E10, 2010

12. Kuo CH, Chang PY, Tu TH, Fay LY, Chang HK, Wu JC, et al: The effect of lumbar lordosis on screw loosening in Dynesys dynamic stabilization: four-year follow-up with computed tomography. BioMed Res Int 2015:152435, 2015

13. Kuo CH, Chang PY, Wu JC, Chang HK, Fay LY, Tu TH, et al: Dynamic stabilization for L4-5 spondylolisthesis: comparison with minimally invasive transforaminal lumbar interbody fusion with more than 2 years of follow-up. Neurosurg Focus 40(1):E3, 2016

14. Lau D, Terman SW, Patel R, La Marca F, Park P: Incidence of and risk factors for superior facet violation in minimally invasive versus open pedicle screw placement during transforaminal lumbar interbody fusion: a comparative analysis. J Neurosurg Spine 18:356-361, 2013

15. Lawhorne TW III, Girardi FP, Mina CA, Pappou I, Cammisa FP Jr: Treatment of degenerative spondylolisthesis: potential impact of dynamic stabilization based on imaging analysis. Eur Spine J 18:815-822, 2009

16. Lee DY, Jung TG, Lee SH: Single-level instrumented miniopen transforaminal lumbar interbody fusion in elderly patients. J Neurosurg Spine 9:137-144, 2008

17. McGirt MJ, Parker SL, Lerner J, Engelhart L, Knight T, Wang MY: Comparative analysis of perioperative surgical site infection after minimally invasive versus open posterior/ transforaminal lumbar interbody fusion: analysis of hospital billing and discharge data from 5170 patients. J Neurosurg Spine 14:771-778, 2011

18. Modic MT, Steinberg PM, Ross JS, Masaryk TJ, Carter JR: Degenerative disk disease: assessment of changes in vertebral body marrow with MR imaging. Radiology 166:193-199, 1988

19. Nakai S, Yoshizawa H, Kobayashi S: Long-term follow-up study of posterior lumbar interbody fusion. J Spinal Disord 12:293-299, 1999

20. Nakashima H, Kawakami N, Tsuji T, Ohara T, Suzuki Y, Saito $\mathrm{T}$, et al: Adjacent segment disease after posterior lumbar interbody fusion: based on cases with a minimum of 10 years of follow-up. Spine (Phila Pa 1976) 40:E831-E841, 2015

21. Park P, Garton HJ, Gala VC, Hoff JT, McGillicuddy JE: Adjacent segment disease after lumbar or lumbosacral fusion: review of the literature. Spine (Phila Pa 1976) 29:19381944, 2004

22. Parker SL, Adogwa O, Witham TF, Aaronson OS, Cheng J, McGirt MJ: Post-operative infection after minimally invasive versus open transforaminal lumbar interbody fusion (TLIF): literature review and cost analysis. Minim Invasive Neurosurg 54:33-37, 2011

23. Payer M, Smoll NR, Oezkan N, Tessitore E: Dynamic transpedicular stabilisation and decompression in single-level degenerative anterolisthesis and stenosis. Acta Neurochir (Wien) 156:221-227, 2014 [Erratum in Acta Neurochir (Wien) 156:759, 2014]

24. Pfirrmann CW, Metzdorf A, Zanetti M, Hodler J, Boos N: Magnetic resonance classification of lumbar intervertebral disc degeneration. Spine (Phila Pa 1976) 26:1873-1878, 2001

25. Poh SY, Yue WM, Chen LT, Guo CM, Yeo W, Tan SB: Twoyear outcomes of transforaminal lumbar interbody fusion. J Orthop Surg (Hong Kong) 19:135-140, 2011

26. Rahm MD, Hall BB: Adjacent-segment degeneration after lumbar fusion with instrumentation: a retrospective study. J Spinal Disord 9:392-400, 1996

27. Riesenburger RI, Safain MG, Ogbuji R, Hayes J, Hwang SW: A novel classification system of lumbar disc degeneration. $\mathbf{J}$ Clin Neurosci 22:346-351, 2015 
28. Schaeren S, Broger I, Jeanneret B: Minimum four-year follow-up of spinal stenosis with degenerative spondylolisthesis treated with decompression and dynamic stabilization. Spine (Phila Pa 1976) 33:E636-E642, 2008

29. Schizas C, Tzinieris N, Tsiridis E, Kosmopoulos V: Minimally invasive versus open transforaminal lumbar interbody fusion: evaluating initial experience. Int Orthop 33:16831688,2009

30. Sengupta DK, Herkowitz HN: Degenerative spondylolisthesis: review of current trends and controversies. Spine (Phila Pa 1976) 30 (6 Suppl):S71-S81, 2005

31. St-Pierre GH, Jack A, Siddiqui MM, Henderson RL, Nataraj A: Nonfusion does not prevent adjacent segment disease: Dynesys long-term outcomes with minimum five-year followup. Spine (Phila Pa 1976) 41:265-273, 2016

32. Sulaiman WA, Singh M: Minimally invasive versus open transforaminal lumbar interbody fusion for degenerative spondylolisthesis grades 1-2: patient-reported clinical outcomes and cost-utility analysis. Ochsner J 14:32-37, 2014

33. Wang J, Zhou Y: Perioperative complications related to minimally invasive transforaminal lumbar fusion: evaluation of 204 operations on lumbar instability at single center. Spine $\mathbf{J}$ 14:2078-2084, 2014

34. Welch WC, Cheng BC, Awad TE, Davis R, Maxwell JH, Delamarter R, et al: Clinical outcomes of the Dynesys dynamic neutralization system: 1-year preliminary results. Neurosurg Focus 22(1):E8, 2007

35. Wimmer C, Gluch H, Krismer M, Ogon M, Jesenko R: APtranslation in the proximal disc adjacent to lumbar spine fusion. A retrospective comparison of mono- and polysegmental fusion in 120 patients. Acta Orthop Scand 68:269-272, 1997

36. Wong AP, Smith ZA, Nixon AT, Lawton CD, Dahdaleh NS,
Wong $\mathrm{RH}$, et al: Intraoperative and perioperative complications in minimally invasive transforaminal lumbar interbody fusion: a review of 513 patients. J Neurosurg Spine 22:487495, 2015

37. Wu JC, Huang WC, Tsai HW, Ko CC, Wu CL, Tu TH, et al: Pedicle screw loosening in dynamic stabilization: incidence, risk, and outcome in 126 patients. Neurosurg Focus 31(4):E9, 2011

38. Yee TJ, Terman SW, La Marca F, Park P: Comparison of adjacent segment disease after minimally invasive or open transforaminal lumbar interbody fusion. J Clin Neurosci 21:1796-1801, 2014

\section{Disclosures}

The authors report no conflict of interest concerning the materials or methods used in this study or the findings specified in this paper.

\section{Author Contributions}

Conception and design: JC Wu. Acquisition of data: Kuo. Analysis and interpretation of data: Kuo, Tu. Drafting the article: Kuo. Critically revising the article: JC Wu. Reviewed submitted version of manuscript: all authors. Approved the final version of the manuscript on behalf of all authors: JC Wu. Administrative/technical/ material support: all authors. Study supervision: Huang, Cheng.

\section{Correspondence}

Jau-Ching Wu: Neurological Institute, Taipei Veterans General Hospital, Taipei, Taiwan. jauching@gmail.com. 